neutral helium, particularly in this low excitation position. These enhanced helium and nitrogen abundances relative to solar or orion values, along with the observed morphology, imply that YM29 belongs to Peimbert's Type I class of planetary nebulae, which presumably originate from more massive progenitors. Our neon abundance determination seems to suffer from the inflation commonly found for low excitation regions in nebulae.

\title{
OPTICALLY DERIVED CARBON ABUNDANCES IN PLANETARY NEBULAE
}

\author{
H.B. French \\ Department of Physics \& Astronomy, University of Oklahoma, USA
}

Gas phase carbon abundances have been determined for a number of bright planetary nebulae from new photoelectric measurements of optical recombination lines (C II $\lambda$ 4267, C III $\lambda 4650$ and C IV $\lambda$ 4659). Because of blending problems for the latter two features, the abundance for any object may have substantial errors, but the average abundance should be reliable. For the twelve best observed planetaries, this average is $\mathrm{C} / \mathrm{H}=(8.4 \pm 2.9 \mathrm{s.d}.) \times 10^{-4}$ by number. If it is assumed that the planetary progenitors had essentially solar abundances, then, based on Cameron's most recent results $\left(\mathrm{C} / \mathrm{H}=4.2 \times 10^{-4}, 0 / \mathrm{H}=6.9 \times 10^{-4}\right)$, it appears that the planetary ejecta have been enriched in carbon, presumably because of dredging of newly synthesized triple $\alpha$ carbon by helium shell flashes during the late evolution of the progenitor. The helium abundance is also slightly high, as would be expected in this interpretation. Since the mean planetary carbon abundance exceeds the solar oxygen abundance, it is possible that the progenitor became a carbon star prior to the ejection of the planetary; that may even have caused the ejection. Because the planetaries in this study were drawn from Peimbert's samples with relatively low mass progenitors ( $1-2.5 \mathrm{M}_{\Theta}$; these are not significantly helium- and nitrogen-rich objects), such a process might be a general feature of late double shell source evolution.

From simple estimates it appears that the contribution to Galactic carbon enrichment from planetaries is comparable to that from supernovae. Because the former represent long-lived stars, and the latter shortlived, we might expect that carbon and oxygen abundances in the Galaxy would not vary in lockstep in the way that, for example, neon and oxygen abundances appear to.

Further observations are underway both to expand the sample of planetaries, and, through higher spectral resolution, to improve the abundances in individual objects. 